\title{
High energy solutions of modified quasilinear fourth-order elliptic equation
}

Xiujuan Wang, Anmin Mao and Aixia Qian*

\section{"Correspondence:} qaixia@amss.ac.cn

School of Mathematical Sciences, Qufu Normal University, Shandong, P.R. China

\section{Springer}

\section{Abstract}

This paper focuses on the following modified quasilinear fourth-order elliptic equation:

$$
\left\{\begin{array}{l}
\Delta^{2} u-\left(a+b \int_{\mathbb{R}^{3}}|\nabla u|^{2} d x\right) \Delta u+\lambda V(x) u-\frac{1}{2} \Delta\left(u^{2}\right) u=f(x, u), \quad \text { in } \mathbb{R}^{3}, \\
u(x) \in H^{2}\left(\mathbb{R}^{3}\right),
\end{array}\right.
$$

where $\Delta^{2}=\Delta(\Delta)$ is the biharmonic operator, $a>0, b \geq 0, \lambda \geq 1$ is a parameter, $V \in C\left(\mathbb{R}^{3}, \mathbb{R}\right), f(x, u) \in C\left(\mathbb{R}^{3} \times \mathbb{R}, \mathbb{R}\right) . V(x)$ and $f(x, u) u$ are both allowed to be sign-changing. Under the weaker assumption $\lim _{|t| \rightarrow \infty} \frac{\int_{0}^{t} f(x, s) d s}{|t|^{3}}=\infty$ uniformly in $x \in \mathbb{R}^{3}$, a sequence of high energy weak solutions for the above problem are obtained.

MSC: $35 J 25 ; 35 J 20 ; 35 J 60 ; 35 J 61$

Keywords: Super-quadratic; High energy solutions; Sign-changing potential; Fountain theorem

\section{Introduction and main results}

In this paper, we consider the following elliptic equation:

$$
\left\{\begin{array}{l}
\triangle^{2} u-\left(a+b \int_{\mathbb{R}^{3}}|\nabla u|^{2} d x\right) \triangle u+\lambda V(x) u-\frac{1}{2} \triangle\left(u^{2}\right) u=f(x, u), \quad \text { in } \mathbb{R}^{3}, \\
u(x) \in H^{2}\left(\mathbb{R}^{3}\right),
\end{array}\right.
$$

where $\Delta^{2}=\Delta(\Delta)$ is the biharmonic operator, the constants $a>0, b \geq 0$, and $\lambda \geq 1$ is a parameter. $V(x): \mathbb{R}^{3} \rightarrow \mathbb{R}$ and $f: \mathbb{R}^{3} \times \mathbb{R} \rightarrow \mathbb{R}$ satisfies the following assumptions:

(V) $V \in C\left(\mathbb{R}^{3}, \mathbb{R}\right), \inf _{\mathbb{R}^{3}} V>-\infty$ and there exists a constant $r>0$ such that

$$
\lim _{|y| \rightarrow \infty} \operatorname{meas}\left\{x \in \mathbb{R}^{3}:|x-y| \leq r, V(x) \leq M\right\}=0, \quad \forall M>0 ;
$$

$\left(F_{1}\right) f \in C\left(\mathbb{R}^{3} \times \mathbb{R}, \mathbb{R}\right)$ and there exists positive constant $C_{0}$ and $p>4$ such that

$$
|f(x, t)| \leq C_{0}\left(|t|+|t|^{p-1}\right), \quad \forall(x, t) \in \mathbb{R}^{3} \times \mathbb{R}
$$

(F) $\lim _{|t| \rightarrow \infty} \frac{F(x, t)}{|t|^{3}}=\infty$ uniformly in $x \in \mathbb{R}^{3}$, where $F(x, t)=\int_{0}^{t} f(x, s) d s$. 
$\left(F_{3}\right)$ There exists a constant $\alpha \geq 0$ such that

$$
f(x, t) t-4 F(x, t) \geq-\alpha t^{2}, \quad \forall(x, t) \in \mathbb{R}^{3} \times \mathbb{R} .
$$

$\left(F_{4}\right) f(x,-t)=-f(x, t)$ for all $(x, t) \in \mathbb{R}^{3} \times \mathbb{R}$.

The Kirchhoff's model considers the changes in length of the string produced by transverse vibrations. It was pointed out in [1-4] that (1.1) models several physical and biological systems where $u$ describes a process which relies on the mean of itself such as the population density. For more mathematical and physical background on Kirchhoff-type problems, we refer the reader to $[1,5-8]$ and the references therein. It is well known that fourth-order elliptic equation has been widely studied since Lazer and Mckenna [9] first proposed to study periodic oscillations and traveling waves in a suspension bridge.

In te recent years, many scholars widely studied the Schrödinger equation under variant assumptions on $V(x)$ and $f(x, u)$, such as [3, 4, 10-13]. In [10], Wu considered the following Schrödinger-Kirchhoff-type problem:

$$
-\left(a+b \int_{\mathbb{R}^{N}}|\nabla u|^{2} d x\right) \Delta u+V(x) u=f(x, u), \quad \text { in } \mathbb{R}^{N}(N \leq 3)
$$

under these hypotheses:

$\left(V^{\prime}\right) V \in C\left(\mathbb{R}^{N}, \mathbb{R}\right)$ satisfies $\inf V(x) \geq a_{1}>0$ and for each $M>0$, meas $\left\{x \in \mathbb{R}^{N}: V(x) \leq\right.$ $M\}<+\infty$, where $a_{1}$ is a constant and meas denotes the Lebesgue measure in $\mathbb{R}^{N}$.

$\left(f_{1}\right) f \in C\left(\mathbb{R}^{N} \times \mathbb{R}, \mathbb{R}\right)$ and $|f(x, t)| \leq C\left(1+|t|^{p-1}\right)$ for some $2 \leq p<2^{*}$, where $C$ is a positive constant;

$\left(f_{2}\right) f(x, t)=o(|t|)$ as $|t| \rightarrow 0$;

$\left(f_{3}\right) \frac{F(x, t)}{t^{4}} \rightarrow+\infty$ as $|t| \rightarrow+\infty$ uniformly in $\forall x \in \mathbb{R}^{N}$;

$\left(f_{4}\right) \operatorname{tf}(x, t) \geq 4 F(x, t), \forall x \in \mathbb{R}^{N}, \forall t \in \mathbb{R}$

Here $\left(f_{3}\right)$ is essential in these references to overcome the missing of compactness. The author got a nontrivial solution of (1.2). In [8], Zhang and Tang also considered the problem (1.2) under the assumption $(V)$, and they obtained infinitely many high energy solutions of the problem (1.2). In [11], Nie studied the following Schrödinger-Kirchhoff-type equation:

$$
\begin{cases}-\left(a+b \int_{\mathbb{R}^{3}}|\nabla u|^{2} d x\right) \Delta u+\lambda V(x) u=f(x, u), & \text { in } \mathbb{R}^{3}, \\ u(x) \rightarrow 0 & \text { as }|x| \rightarrow \infty\end{cases}
$$

under the assumption $\left(V^{\prime}\right)$. They got a sequence of high energy weak solutions whenever $\lambda>0$ is sufficiently large. In [14], $\mathrm{Xu}$ and Chen also used condition $\left(V^{\prime}\right)$ to study the problem (1.3).

More recently, Cheng and Tang [15] studied the following elliptic equation:

$$
\left\{\begin{array}{l}
\triangle^{2} u-\Delta u+V(x) u-\frac{1}{2} \triangle\left(u^{2}\right) u=f(x, u), \quad \text { in } \mathbb{R}^{N} \\
u(x) \in H^{2}\left(\mathbb{R}^{N}\right),
\end{array}\right.
$$

under the assumption $\left(f_{3}\right)$. Clearly, the problem (1.1) is equivalent to (1.4) whenever $N=3$, $a=1, b=0, \lambda=1$, and condition $\left(f_{3}\right)$ is stronger than $\left(F_{2}\right)$. 
Motivated by the work we discussed above, we will use weaker conditions $\left(F_{2}\right),\left(F_{3}\right)$ instead of the common assumptions $\left(f_{3}\right),\left(f_{4}\right)$, while $V(x)$ and $f(x, u) u$ are both allowed to be sign-changing. We will further study and establish the existence of infinitely many high energy solutions of (1.1) whenever $\lambda \geq 1$, by using the fountain theorem $[16,17]$ or its other versions $[18,19]$. To the best of our knowledge, there is little work concerning this case up to now.

The following are our main results.

Theorem 1.1 Assume that $(V)$ and $\left(F_{1}\right)-\left(F_{4}\right)$ are satisfied, then problem (1.1) possesses infinitely many high energy solutions whenever $\lambda \geq 1$.

Corollary 1.2 Assume that $(V)$ and $\left(F_{1}\right)-\left(F_{4}\right)$ are satisfied, then problem (1.3) possesses infinitely many high energy nontrivial solutions whenever $\lambda \geq 1$.

Remark 1.3 Obviously, the condition $(V)$ is weaker than $\left(V^{\prime}\right) ;\left(F_{1}\right)$ is weaker than $\left(f_{1}\right)$ and $\left(f_{2}\right) ;\left(F_{3}\right)$ is weaker than $\left(f_{7}\right)[14]$ and $\left(f_{4}\right) ;\left(F_{2}\right)$ is weaker than $\left(g_{2}\right)$ [15]. Furthermore, we do not require $\lambda$ large enough, but we only need $\lambda \geq 1$. Therefore, our results extend and improve Theorem 1 [10], Theorem 1.2 [11], Theorem 1.3 [14], Theorem 1.1 [8], Theorem 1.4 [15] and so on.

Remark 1.4 There are many functions satisfying assumptions $\left(F_{1}\right)-\left(F_{4}\right)$ not $\left(f_{3}\right)$. For example

$$
f(x, u)=4 u^{3}-\frac{2 u\left(1+u^{2}\right) \ln \left(1+u^{2}\right)+2 u^{3}-2 u^{3} \ln \left(1+u^{2}\right)}{\left(1+u^{2}\right)^{2}}
$$

for all $(x, u) \in \mathbb{R}^{3} \times \mathbb{R}$.

Indeed, $F(x, u)=u^{4}-\frac{u^{2} \ln \left(1+u^{2}\right)}{1+u^{2}}$, then we can find a positive constant $\alpha$ such that

$$
f(x, u) u-4 F(x, u)+\alpha u^{2}=\frac{2 u^{2} \ln \left(1+u^{2}-2 u^{4}+\alpha u^{6}+2 \alpha u^{4}+\alpha u^{2}\right)}{\left(1+u^{2}\right)^{2}} \geq 0 .
$$

\section{Preliminary lemmas and proof of our main result}

In order to apply the variational method, we first recall some related preliminaries and establish a corresponding variational framework for our problem (1.1); then we give the proof of Theorem 1.1.

For $1<s<+\infty$, define the Sobolev space

$$
W^{m, s}\left(\mathbb{R}^{N}\right)=\left\{u \in L^{s}\left(\mathbb{R}^{N}\right)\left|D^{\alpha} u \in L^{s}\left(\mathbb{R}^{N}\right),\right| \alpha \mid \leq m\right\}
$$

equipped with the norm

$$
\|u\|_{W^{m, s}\left(\mathbb{R}^{N}\right)}=\left(\sum_{|\alpha| \leq m} \int_{\mathbb{R}^{N}}\left|D^{\alpha} u\right|^{s} d x\right)^{\frac{1}{s}}
$$


where $\alpha=\left(\alpha_{1}, \alpha_{2}, \ldots, \alpha_{N}\right)$ with $\alpha_{i} \in \mathbb{Z}^{+}$(the set of all non-negative integers), $i=1,2, \ldots, N$, $|\alpha|=\alpha_{1}+\alpha_{2}+\cdots+\alpha_{N}$ and

$$
D^{\alpha} u=\frac{\partial^{|\alpha|} u}{\partial x_{1}^{\alpha_{1}} \partial x_{2}^{\alpha_{2}} \cdots \partial x_{N}^{\alpha_{N}}}
$$

For $s=2, H^{m}\left(\mathbb{R}^{N}\right)=W^{m, 2}\left(\mathbb{R}^{N}\right)$ is a Hilbert space equipped with the scalar product

$$
\langle u, v\rangle_{H^{m}}=\sum_{|\alpha| \leq m} \int_{\mathbb{R}^{N}} D^{\alpha} u D^{\alpha} v d x
$$

and the norm

$$
\|u\|_{H^{m}}=\langle u, u\rangle_{H^{m}}^{\frac{1}{2}}=\left(\sum_{|\alpha| \leq m} \int_{\mathbb{R}^{N}}\left|D^{\alpha} u\right|^{2} d x\right)^{\frac{1}{2}} .
$$

Moreover, for $m=2$ one has

$$
\begin{aligned}
& \langle u, v\rangle_{H^{2}}=\int_{\mathbb{R}^{N}}(\Delta u \Delta v+\nabla u \nabla v+u v) d x, \\
& \|u\|_{H^{2}}^{2}=\langle u, v\rangle_{H^{2}}=\int_{\mathbb{R}^{N}}\left(|\Delta u|^{2}+|\nabla u|^{2}+u^{2}\right) d x,
\end{aligned}
$$

whenever $u, v \in H^{2}\left(\mathbb{R}^{N}\right)$.

Under assumption $(V)$, we can find $V_{0} \geq 0$ such that $\widetilde{V}(x)=V(x)+V_{0} \geq 1$ for all $x \in \mathbb{R}^{3}$. Then

$$
E_{\lambda}=\left\{u \in H^{2}\left(\mathbb{R}^{3}\right): \int_{\mathbb{R}^{3}}\left(a|\nabla u|^{2}+\lambda \tilde{V}(x) u^{2}\right) d x<\infty\right\}
$$

is a Hilbert space endowed with the norm

$$
\|u\|_{\lambda}=\left(\int_{\mathbb{R}^{3}}\left(|\Delta u|^{2}+a|\nabla u|^{2}+\lambda \widetilde{V}(x) u^{2}\right) d x\right)^{\frac{1}{2}}
$$

Let

$$
\begin{aligned}
\Phi_{\lambda}(u)= & \frac{1}{2} \int_{\mathbb{R}^{3}}\left(|\Delta u|^{2}+a|\nabla u|^{2}+\lambda V(x) u^{2}\right) d x+\frac{b}{4}\left(\int_{\mathbb{R}^{3}}|\nabla u|^{2} d x\right)^{2} \\
& +\frac{1}{2} \int_{\mathbb{R}^{3}} u^{2}|\nabla u|^{2} d x-\int_{\mathbb{R}^{3}} F(x, u) d x, \quad \forall u \in E_{\lambda} .
\end{aligned}
$$

By condition $(V),\left(F_{1}\right)$ and the fact $\int_{\mathbb{R}^{3}} u^{2}|\nabla u|^{2} d x<\infty$ (see Lemma 2.2 in [20]), $\Phi_{\lambda}$ is a well-defined class $C^{1}$ functional. For all $u, v \in E_{\lambda}$

$$
\begin{aligned}
\left\langle\Phi_{\lambda}^{\prime}(u), v\right\rangle= & \int_{\mathbb{R}^{3}}(\Delta u \Delta v+a \nabla u \nabla v+\lambda V(x) u v) d x+b \int_{\mathbb{R}^{3}}|\nabla u|^{2} d x \int_{\mathbb{R}^{3}} \nabla u \nabla v d x \\
& +\int_{\mathbb{R}^{3}}\left(u v|\nabla u|^{2}+u^{2} \nabla u \nabla v\right) d x-\int_{\mathbb{R}^{3}} f(x, u) v d x .
\end{aligned}
$$


Clearly, seeking a weak solution of problem (1.1) is equivalent to finding a critical point of the functional $\Phi_{\lambda}$.

Definition 2.1 A sequence $\left\{u_{n}\right\} \subset E_{\lambda}$ is said to be a $(C)_{c}$ sequence if

$$
\Phi_{\lambda}\left(u_{n}\right) \rightarrow c, \quad\left\|\Phi_{\lambda}^{\prime}\left(u_{n}\right)\right\|_{\lambda}\left(1+\left\|u_{n}\right\|_{\lambda}\right) \rightarrow 0 .
$$

$\Phi_{\lambda}$ is said to satisfy the $(C)_{c}$ condition if any $(C)_{c}$ sequence possesses a convergent subsequence.

Let $E_{\lambda}^{\prime}=\left\{u \in H^{2}\left(\mathbb{R}^{N}\right): \int_{\mathbb{R}^{N}}\left(a|\nabla u|^{2}+\lambda \widetilde{V}(x) u^{2}\right) d x<\infty\right\}$.

Lemma 2.2 Under assumption $(V)$, the embedding $E_{\lambda}^{\prime} \hookrightarrow L^{s}\left(\mathbb{R}^{N}\right)$ is compact for $2 \leq s<2$, where $2_{*}=\frac{2 N}{N-4}$, if $N>4 ; 2_{*}=+\infty$, if $N \leq 4$.

Proof Define

$$
E=\left\{u \in H^{1}\left(\mathbb{R}^{N}\right): \int_{\mathbb{R}^{N}}\left(a|\nabla u|^{2}+\lambda \widetilde{V}(x) u^{2}\right) d x<\infty\right\} .
$$

By Propositions 3.1 and 3.3 in [13], we know that the embedding $E \hookrightarrow L^{s}\left(\mathbb{R}^{N}\right)$ is compact for $2 \leq s<2 *$ due to the condition $(V)$, and the embedding $E_{\lambda}^{\prime} \hookrightarrow E$ is continuous, therefore, the embedding $E_{\lambda}^{\prime} \hookrightarrow L^{s}\left(\mathbb{R}^{N}\right)$ is compact for $2 \leq s<2 *$.

Lemma 2.3 Under assumptions $(V),\left(F_{1}\right)$, any bounded $(C)_{c}$ sequence of $\Phi_{\lambda}$ has a strongly convergent subsequence in $E_{\lambda}$.

Proof Let $\left\{u_{n}\right\} \subset E_{\lambda}$ hold with

$$
\sup _{n}\left\|u_{n}\right\|_{\lambda}<+\infty
$$

Then up to a subsequence, there exists a constant $c \in \mathbb{R}$ such that

$$
\Phi_{\lambda}\left(u_{n}\right) \rightarrow c, \quad \Phi_{\lambda}^{\prime}\left(u_{n}\right) \rightarrow 0
$$

According to Lemma 2.2, going if necessary to a subsequence, we can assume that there exists $u \in E_{\lambda}$ such that

$$
\begin{array}{ll}
u_{n} \rightarrow u & \text { in } E_{\lambda}, \\
u_{n} \rightarrow u & \text { in } L^{s}\left(\mathbb{R}^{3}\right)(2 \leq s<+\infty), \\
u_{n} \rightarrow u & \text { a.e. in } \mathbb{R}^{3} .
\end{array}
$$

By an elementary computation,

$$
\begin{aligned}
& \left\langle\Phi_{\lambda}^{\prime}\left(u_{n}\right)-\Phi^{\prime}(u), u_{n}-u\right\rangle \\
& \quad \geq\left\|u_{n}-u\right\|_{\lambda}^{2}-\lambda V_{0} \int_{\mathbb{R}^{3}}\left|u_{n}-u\right|^{2} d x
\end{aligned}
$$


Wang et al. Boundary Value Problems (2018) 2018:54

Page 6 of 13

$$
\begin{aligned}
& +b\left(\int_{\mathbb{R}^{3}}\left|\nabla u_{n}\right|^{2} d x-\int_{\mathbb{R}^{3}}|\nabla u|^{2} d x\right) \int_{\mathbb{R}^{3}} \nabla u_{n} \nabla\left(u_{n}-u\right) d x \\
& +\int_{\mathbb{R}^{3}}\left(u_{n}\left|\nabla u_{n}\right|^{2}-u|\nabla u|^{2}\right)\left(u_{n}-u\right) d x+\int_{\mathbb{R}^{3}}\left(u_{n}^{2}-u^{2}\right) \nabla u \nabla\left(u_{n}-u\right) d x \\
& +\int_{\mathbb{R}^{3}}\left(f(x, u)-f\left(x, u_{n}\right)\right)\left(u_{n}-u\right) d x .
\end{aligned}
$$

Clearly, $\lambda V_{0} \int_{\mathbb{R}^{3}}\left|u_{n}-u\right|^{2} d x \rightarrow 0$, and $\left\langle\Phi_{\lambda}^{\prime}\left(u_{n}\right)-\Phi^{\prime}(u), u_{n}-u\right\rangle \rightarrow 0$. Then, since $\left\{u_{n}\right\} \subset E_{\lambda}$ is bounded, we have

$$
\begin{aligned}
& \left|b\left(\int_{\mathbb{R}^{3}}\left|\nabla u_{n}\right|^{2} d x-\int_{\mathbb{R}^{3}}|\nabla u|^{2} d x\right) \int_{\mathbb{R}^{3}} \nabla u_{n} \nabla\left(u_{n}-u\right) d x\right| \\
& \leq\left|b\left(\int_{\mathbb{R}^{3}}\left|\nabla u_{n}\right|^{2} d x-\int_{\mathbb{R}^{3}}|\nabla u|^{2} d x\right) \int_{\mathbb{R}^{3}} \nabla u \nabla\left(u_{n}-u\right) d x\right| \\
& \quad+\left.\left|b\left(\int_{\mathbb{R}^{3}}\left|\nabla u_{n}\right|^{2} d x-\int_{\mathbb{R}^{3}}|\nabla u|^{2} d x\right) \int_{\mathbb{R}^{3}}\right| \nabla\left(u_{n}-u\right)\right|^{2} d x \mid \\
& \quad \rightarrow 0 .
\end{aligned}
$$

Note that $E_{\lambda} \hookrightarrow H^{2}\left(\mathbb{R}^{3}\right) \hookrightarrow W^{1, s}\left(\mathbb{R}^{3}\right)$ for $2 \leq s \leq+\infty$,

$$
\begin{aligned}
\int_{\mathbb{R}^{3}}\left|\nabla u_{n}\right|^{3} d x & \leq \int_{\mathbb{R}^{3}}\left(\left|u_{n}\right|^{2}+\sum_{i=1}^{3}\left|\frac{\partial u_{n}}{\partial x_{i}}\right|^{2}\right)^{\frac{3}{2}} d x \\
& \leq \int_{\mathbb{R}^{3}}\left(\left|u_{n}\right|+\sum_{i=1}^{3}\left|\frac{\partial u_{n}}{\partial x_{i}}\right|\right)^{3} d x \\
& \leq \int_{\mathbb{R}^{3}}\left[4 \max \left\{\left|u_{n}\right|,\left|\frac{\partial u_{n}}{\partial x_{1}}\right|,\left|\frac{\partial u_{n}}{\partial x_{2}}\right|,\left|\frac{\partial u_{n}}{\partial x_{3}}\right|\right\}\right]^{3} d x \\
& \leq 4^{3} \int_{\mathbb{R}^{3}}\left(\left|u_{n}\right|^{3}+\sum_{i=1}^{3}\left|\frac{\partial u_{n}}{\partial x_{i}}\right|^{3}\right) d x \\
& =4^{3}\left\|u_{n}\right\|_{W^{1,3}\left(\mathbb{R}^{3}\right)}^{3} \\
& \leq 4^{3} S_{3}^{3}\left\|u_{n}\right\|_{\lambda}^{3},
\end{aligned}
$$

where

$$
S_{s}=\sup _{u \in E_{\lambda},\|u\|_{\lambda}=1}\|u\|_{W^{1, s}}, \quad \forall 2 \leq s \leq+\infty
$$

Applying (2.3)-(2.5) and (2.8), there exist constants $C_{1}>0$ such that

$$
\begin{aligned}
& \left|\int_{\mathbb{R}^{3}}\left(u_{n}\left|\nabla u_{n}\right|^{2}-u|\nabla u|^{2}\right)\left(u_{n}-u\right) d x\right| \\
& \quad \leq \int_{\mathbb{R}^{3}}\left|u_{n}\right|\left|\nabla u_{n}\right|^{2}\left|u_{n}-u\right| d x+\int_{\mathbb{R}^{3}}|u||\nabla u|^{2}\left|u_{n}-u\right| d x \\
& \quad \leq\left(\int_{\mathbb{R}^{3}}\left|u_{n}\right|^{6} d x\right)^{\frac{1}{6}}\left(\int_{\mathbb{R}^{3}}\left|\nabla u_{n}\right|^{3} d x\right)^{\frac{2}{3}}\left(\int_{\mathbb{R}^{3}}\left|u_{n}-u\right|^{6} d x\right)^{\frac{1}{6}}
\end{aligned}
$$




$$
\begin{aligned}
& \quad+\left(\int_{\mathbb{R}^{3}}|u|^{6} d x\right)^{\frac{1}{6}}\left(\int_{\mathbb{R}^{3}}|\nabla u|^{3} d x\right)^{\frac{2}{3}}\left(\int_{\mathbb{R}^{3}}\left|u_{n}-u\right|^{6} d x\right)^{\frac{1}{6}} \\
& \leq C_{1}\left\|u_{n}-u\right\|_{L^{6}} \rightarrow 0, \quad \text { as } n \rightarrow \infty,
\end{aligned}
$$

and $C_{1}^{\prime}>0$ such that

$$
\begin{aligned}
& \left|\int_{\mathbb{R}^{3}}\left(u_{n}^{2}-u^{2}\right) \nabla u \nabla\left(u_{n}-u\right) d x\right| \\
& \quad \leq \int_{\mathbb{R}^{3}}\left|u_{n}-u\right|\left|u_{n}+u\right||\nabla u|\left|\nabla\left(u_{n}-u\right)\right| d x \\
& \quad \leq\left(\int_{\mathbb{R}^{3}}\left|u_{n}-u\right|^{6}\right)^{\frac{1}{6}}\left(\int_{\mathbb{R}^{3}}\left|u_{n}+u\right|^{6}\right)^{\frac{1}{6}}\left(\int_{\mathbb{R}^{3}}|\nabla u|^{3}\right)^{\frac{1}{3}}\left(\int_{\mathbb{R}^{3}}\left|\nabla\left(u_{n}-u\right)\right|^{3}\right)^{\frac{1}{3}} \\
& \quad \leq C_{1}^{\prime}\left\|u_{n}-u\right\|_{L^{6}} \rightarrow 0, \quad \text { as } n \rightarrow \infty .
\end{aligned}
$$

By $\left(F_{1}\right)$ and the Hölder inequality,

$$
\begin{aligned}
& \left|\int_{\mathbb{R}^{3}}\left(f(x, u)-f\left(x, u_{n}\right)\right)\left(u_{n}-u\right) d x\right| \\
& \quad \leq C_{0} \int_{\mathbb{R}^{3}}\left[|u|+|u|^{p-1}+\left|u_{n}\right|+\left|u_{n}\right|^{p-1}\right]\left|u_{n}-u\right| d x \\
& \quad \leq C_{0}\left[\left(\left\|u_{n}\right\|_{L^{2}}+\|u\|_{L^{2}}\right)\left\|u_{n}-u\right\|_{L^{2}}+\left(\left\|u_{n}\right\|_{L^{p}}^{p-1}+\|u\|_{L^{p}}^{p-1}\right)\left\|u_{n}-u\right\|_{L^{p}}\right] .
\end{aligned}
$$

Then, combining the last inequality with (2.5), we get

$$
\int_{\mathbb{R}^{3}}\left(f(x, u)-f\left(x, u_{n}\right)\right)\left(u_{n}-u\right) d x \rightarrow 0, \quad \text { as } n \rightarrow \infty .
$$

Hence, the combination of (2.7) and (2.9)-(2.11) implies that

$$
u_{n} \rightarrow u \quad \text { in } E_{\lambda} .
$$

Therefore, the proof is complete.

Lemma 2.4 Assume that $(V)$ and $\left(F_{1}\right)-\left(F_{3}\right)$ hold, then $\Phi_{\lambda}$ satisfies the $(C)_{c}$ condition.

Proof Let $\left\{u_{n}\right\} \subset E_{\lambda}$ be such that

$$
\Phi_{\lambda}\left(u_{n}\right) \rightarrow c, \quad\left\|\Phi_{\lambda}^{\prime}\left(u_{n}\right)\right\|_{\lambda}\left(1+\left\|u_{n}\right\|_{\lambda}\right) \rightarrow 0 .
$$

First, we prove that $\left\{u_{n}\right\}$ is bounded in $E_{\lambda}$. By $\left(F_{3}\right),(2.1),(2.2)$ and (2.12), one has

$$
\begin{aligned}
c+o(1)= & \Phi_{\lambda}\left(u_{n}\right)-\frac{1}{4}\left\langle\Phi_{\lambda}^{\prime}\left(u_{n}\right), u_{n}\right\rangle \\
= & \frac{1}{4} \int_{\mathbb{R}^{3}}\left(\left|\Delta u_{n}\right|^{2}+a\left|\nabla u_{n}\right|^{2}+\lambda \widetilde{V}(x) u_{n}^{2}\right) d x \\
& +\int_{\mathbb{R}^{3}}\left[\frac{1}{4} f\left(x, u_{n}\right) u_{n}-F\left(x, u_{n}\right)-\frac{\lambda}{4} V_{0} u_{n}^{2}\right] d x
\end{aligned}
$$




$$
\geq \frac{1}{4}\left\|u_{n}\right\|_{\lambda}^{2}-\frac{\alpha+\lambda V_{0}}{4} \int_{\mathbb{R}^{3}} u_{n}^{2} d x
$$

Thus, it remains to show that $\left\{u_{n}\right\}$ is bounded in $L^{2}\left(\mathbb{R}^{3}\right)$. Otherwise, suppose that $\left\|u_{n}\right\|_{2} \rightarrow$ $\infty$ and then $\left\|u_{n}\right\|_{\lambda} \rightarrow \infty$. Let $\omega_{n}=\frac{u_{n}}{\left\|u_{n}\right\|_{\lambda}}$, then $\left\|\omega_{n}\right\|_{\lambda}=1$. According to Lemma 2.2, up to a subsequence, for some $\omega \in E_{\lambda}$, we obtain

$$
\begin{array}{ll}
\omega_{n} \rightarrow \omega & \text { in } E_{\lambda}, \\
\omega_{n} \rightarrow \omega & \text { in } L^{2}\left(\mathbb{R}^{3}\right), \\
\omega_{n} \rightarrow \omega & \text { a.e. in } \mathbb{R}^{3} .
\end{array}
$$

Clearly, we deduce that $\omega \neq 0$ from (2.13). Then, for $x \in\left\{y \in \mathbb{R}^{3}: \omega(y) \neq 0\right\}$, we have $\left|u_{n}(x)\right| \rightarrow \infty$ as $n \rightarrow \infty$. For any given $u \in H^{2}\left(\mathbb{R}^{3}\right) \backslash\{0\}$, define

$$
\begin{aligned}
g(t) & =\left\|t^{-1} u(t x)\right\|_{H^{2}}^{2}-1 \\
& =\frac{1}{t} \int_{\mathbb{R}^{3}}|\Delta u|^{2} d x+\frac{1}{t^{3}} \int_{\mathbb{R}^{3}}|\nabla u|^{2} d x+\frac{1}{t^{5}} \int_{\mathbb{R}^{3}} u^{2} d x-1, \quad \forall t>0 .
\end{aligned}
$$

By an elementary computation, there exists a unique $T=\widetilde{t}(u)>0$ such that

$$
g(T)=0, \quad \forall u \in H^{2}\left(\mathbb{R}^{3}\right) \backslash\{0\} .
$$

This implies that $g(t)=0$ defines a functional $T=\widetilde{t}(u)$ on $H^{2}\left(\mathbb{R}^{3}\right) \backslash\{0\}$. We define $\tilde{t}(0)=0$. It is easy to verify that $T=\widetilde{t}(u)$ is continuous and $\widetilde{t}(u) \rightarrow \infty$ as $\|u\|_{H^{2}} \rightarrow \infty$.

Due to the definition of $g$, for any $u \in H^{2}\left(\mathbb{R}^{3}\right) \backslash\{0\}$, there exists

$$
v(x)=T^{-1} u(T x) \in H^{2}\left(\mathbb{R}^{3}\right)
$$

such that

$$
\|v\|_{H^{2}}=1
$$

Note that $u_{n} \neq 0$ for large $n \in \mathbb{N}$, then there exist

$$
v_{n}(x)=T_{n}^{-1} u_{n}\left(T_{n} x\right) \in H^{2}\left(\mathbb{R}^{3}\right)
$$

such that

$$
\left\|v_{n}\right\|_{H^{2}}=1
$$

That is,

$$
u_{n}(x)=T_{n} v_{n}\left(T_{n}^{-1} x\right)
$$

with $\left\|v_{n}\right\|_{H^{2}}=1$ for large $n \in \mathbb{N}$. Moreover, we have

$$
T_{n}=\widetilde{t}\left(u_{n}\right) \rightarrow \infty \quad \text { as } n \rightarrow \infty
$$


and

$$
\left\{x \in \mathbb{R}^{3}: v_{n}(x) \neq 0\right\} \neq \emptyset \quad \text { for large } n \in \mathbb{N} \text {. }
$$

From $\left(F_{1}\right)-\left(F_{3}\right)$, there are $R_{0}>0$ and $C_{2}>0$ such that, for all $x \in \mathbb{R}^{3}$,

$$
f(x, u) u+\alpha u^{2} \geq 4 F(x, u) \geq 0, \quad \forall|u| \geq R_{0},
$$

and

$$
|f(x, u) u| \leq C_{2} u^{2}, \quad \forall|u| \leq R_{0}
$$

Thus, by $\left(F_{3}\right),(2.1),(2.2),(2.12)-(2.15)$ and $\left\|v_{n}\right\|_{H^{2}}=1$,

$$
\begin{aligned}
c+o(1)= & \Phi_{\lambda}\left(u_{n}\right)-\frac{1}{2}\left\langle\Phi_{\lambda}^{\prime}\left(u_{n}\right), u_{n}\right\rangle \\
\geq & -\frac{b}{4}\left\|\nabla u_{n}\right\|_{2}^{4}-\frac{\alpha}{4} \int_{\mathbb{R}^{3}} u_{n}^{2} d x-\frac{1}{2} \int_{\mathbb{R}^{3}} u_{n}^{2}\left|\nabla u_{n}\right|^{2} d x+\frac{1}{4} \int_{\mathbb{R}^{3}} f\left(x, u_{n}\right) u_{n} d x \\
= & -\frac{b T_{n}^{6}}{4}\left\|\nabla v_{n}\right\|_{2}^{4}-\frac{\alpha T_{n}^{5}}{4} \int_{\mathbb{R}^{3}} v_{n}^{2} d x-\frac{T_{n}^{5}}{2} \int_{\mathbb{R}^{3}} v_{n}^{2}\left|\nabla v_{n}\right|^{2} d x \\
& +\frac{T_{n}^{3}}{4} \int_{\left|T_{n} v_{n}\right| \leq R_{0}} f\left(T_{n} x, T_{n} v_{n}\right) T_{n} v_{n} d x+\frac{T_{n}^{6}}{4} \int_{\left|T_{n} v_{n}\right| \geq R_{0}} \frac{f\left(T_{n} x, T_{n} v_{n}\right) T_{n} v_{n}}{T_{n}^{3}} d x \\
\geq & \frac{T_{n}^{6}}{4}\left\{-b-\frac{\alpha+C_{2}}{T_{n}}+\int_{\left|T_{n} v_{n}\right| \geq R_{0}} \frac{f\left(T_{n} x, T_{n} v_{n}\right) T_{n} v_{n}}{T_{n}^{3}} d x\right. \\
& \left.-\frac{2 \int_{\mathbb{R}^{3}} v_{n}^{2}\left|\nabla v_{n}\right|^{2} d x}{T_{n}}\right\} .
\end{aligned}
$$

By the Hölder inequality and the Sobolev embedding inequality, we see that the sequence of integrals $\int_{\mathbb{R}^{3}} v_{n}^{2}\left|\nabla v_{n}\right|^{2} d x<\infty$, since $\left\|v_{n}\right\|_{H^{2}}=1$; on the other hand, by $\left(F_{2}\right)$ and (2.14), we have

$$
\int_{\left|T_{n} v_{n}\right| \geq R_{0}} \frac{f\left(T_{n} x, T_{n} v_{n}\right) T_{n} v_{n}}{T_{n}^{3}} d x \rightarrow+\infty \quad \text { as } n \rightarrow+\infty,
$$

which contradicts (2.16). Hence, $\left\{u_{n}\right\}$ is bounded in $L^{2}\left(\mathbb{R}^{3}\right)$. This shows that $\left\{u_{n}\right\}$ is bounded in $E_{\lambda}$ due to (2.13). By Lemma 2.3, $\left\{u_{n}\right\}$ contains a convergent subsequence.

Next, we define

$$
X_{j}=\mathbb{R} e_{j}, \quad Y_{k}=\bigoplus_{j=1}^{k} X_{j}, \quad Z_{k}=\overline{\bigoplus_{j=k+1}^{\infty} X_{j}}, \quad k \in \mathbb{Z},
$$

where $\left\{e_{j}\right\}$ is an orthonormal basis of $E_{\lambda}$.

Lemma 2.5 Assume that $(V)$ holds, then, for $2 \leq s<2_{*}$,

$$
\beta_{k}(s)=\sup _{u \in Z_{k},\|u\|_{\lambda}=1}\|u\|_{s} \rightarrow 0, \quad k \rightarrow \infty .
$$


Proof By virtue of Lemma 2.2, we can prove the conclusion in a similar way to [16, Lemma 3.8] and [17, Corollary 8.18].

Lemma 2.6 Assume that $(V)$ and $\left(F_{1}\right)$ hold, then there exist constants $\rho, \alpha>0$ such that $\left.\Phi\right|_{\partial B_{\rho} \cap Z_{m}} \geq \alpha$.

Proof From (2.1) and $\left(F_{1}\right)$, for all $u \in E_{\lambda}$ we have

$$
\begin{aligned}
\Phi_{\lambda}(u)= & \frac{1}{2} \int_{\mathbb{R}^{3}}\left(|\Delta u|^{2}+a|\nabla u|^{2}+\lambda V(x) u^{2}\right) d x+\frac{b}{4}\left(\int_{\mathbb{R}^{3}}|\nabla u|^{2} d x\right)^{2} \\
& +\frac{1}{2} \int_{\mathbb{R}^{3}} u^{2}|\nabla u|^{2} d x-\int_{\mathbb{R}^{3}} F(x, u) d x \\
\geq & \frac{1}{2}\|u\|_{\lambda}^{2}-\left(\frac{\lambda V_{0}+C_{0}}{2}\|u\|_{2}^{2}+\frac{C_{0}}{p}\|u\|_{p}^{p}\right) .
\end{aligned}
$$

By virtue of Lemma 2.5 , we can choose an integer $m \geq 1$, for all $u \in Z_{m}$, satisfying

$$
\begin{aligned}
\|u\|_{2}^{2} & \leq \frac{1}{2\left(\lambda V_{0}+C_{0}\right)}\|u\|_{\lambda}^{2}, \\
\|u\|_{p}^{p} & \leq \frac{p}{4 C_{0}}\|u\|_{\lambda}^{p} .
\end{aligned}
$$

Combining this with (2.17), one has

$$
\Phi_{\lambda}(u) \geq \frac{1}{4}\|u\|_{\lambda}^{2}\left(1-\|u\|_{\lambda}^{p-2}\right)
$$

Note that, if we let $\rho=\|u\|_{\lambda}>0$ be sufficiently small, then $\Phi_{\lambda}(u) \geq \frac{1}{8} \rho^{2}>0$.

Lemma 2.7 Assume that $(V),\left(F_{1}\right)$ and $\left(F_{2}\right)$ hold, then, for any finite dimensional subspace $E \subset E_{\lambda}$, there exists $R=R(E)>0$ such that $\left.\Phi_{\lambda}\right|_{E \backslash B_{\rho}}<0$.

Proof According to the proof of Lemma 2.4, we know that, for any $u \in E \backslash\{0\}$, there exists a unique $T=\widetilde{t}(u)>0$ such that

$$
v(x)=T^{-1} u(T x) \in H^{2}\left(\mathbb{R}^{3}\right) \text { and }\|v\|_{H^{2}}=1 .
$$

Hence

$$
u(x)=T v\left(T^{-1} x\right) \quad \text { with }\|v\|_{H^{2}}=1 \text { and } T>0 .
$$

By the equivalence of norms in the finite dimensional space $E$, there exists $C_{3}>0$ such that

$$
\min \{a, 1\}\|u\|_{H^{2}}^{2} \leq\|u\|_{\lambda}^{2} \leq C_{3}\|u\|_{2}^{2}
$$

Combining this with

$$
T=\widetilde{t}(u) \rightarrow \infty \quad \text { as }\|u\|_{\lambda} \rightarrow \infty \text { uniformly in } E,
$$


we find that, for any $\delta>0$, there exists a large $R=R(E, \delta)>0$ such that

$$
T=\widetilde{t}(u) \geq \delta \quad \text { for all } u \in E \text { with }\|u\|_{\lambda} \geq R .
$$

By $\left(F_{1}\right)$, there exists $C_{4}>0$, for all $x \in \mathbb{R}^{N},|u| \leq R_{0}$ such that

$$
|F(x, u)| \leq C_{4} u^{2}
$$

where $R_{0}$ is given by (2.15). Combining (2.1) with $\|v\|_{H^{2}}=1$, it follows that for all $u \in E \backslash\{0\}$

$$
\begin{aligned}
\Phi_{\lambda}(u) & =\frac{1}{2}\|u\|_{\lambda}^{2}+\frac{b}{4}\|\nabla u\|_{2}^{4}+\frac{1}{2} \int_{\mathbb{R}^{3}} u^{2}|\nabla u|^{2} d x-\int_{\mathbb{R}^{3}}\left[\frac{\lambda V_{0}}{2} u^{2}+F(x, u)\right] d x \\
& \leq \frac{C_{3}}{2}\|u\|_{2}^{2}+\frac{b}{4}\|\nabla u\|_{2}^{4}+\frac{1}{2} \int_{\mathbb{R}^{3}} u^{2}|\nabla u|^{2} d x-\int_{\mathbb{R}^{3}}\left[\frac{\lambda V_{0}}{2} u^{2}+F(x, u)\right] d x \\
& =\frac{C_{3}-\lambda V_{0}}{2} T^{5}\|v\|_{2}^{2}+\frac{b T^{6}}{4}\|\nabla v\|_{2}^{4}-T^{3} \int_{\mathbb{R}^{3}} F(T x, T v) d x+\frac{1}{2} \int_{\mathbb{R}^{3}} u^{2}|\nabla u|^{2} d x \\
& \leq T^{6}\left(\frac{b}{4}+\frac{C_{3}+\lambda V_{0}+2 C_{4}}{2 T}-\int_{|T v| \geq R_{0}} \frac{F(T x, T v)}{T^{3}} d x\right)+\frac{1}{2} \int_{\mathbb{R}^{3}} u^{2}|\nabla u|^{2} d x \\
& =\Psi(T) .
\end{aligned}
$$

Note that $v \neq 0$, then it follows from $\left(F_{2}\right)$ that

$$
\frac{F(T x, T v)}{|T v|^{3}} \rightarrow+\infty \quad \text { as } T \rightarrow+\infty .
$$

Thus

$$
\int_{|T v| \geq R_{0}} \frac{F(T x, T v)}{T^{3}} \rightarrow+\infty \quad \text { as } T \rightarrow+\infty .
$$

Combining this with (2.18), we obtain

$$
\Psi(T) \rightarrow-\infty \quad \text { as } T \rightarrow+\infty .
$$

Thus, there exists a large $T_{0}>0$ such that

$$
\Psi(T) \leq-1
$$

for all $T \geq T_{0}$. Taking $\delta=T_{0}$, then there exists a large $R=R(E)>0$ such that

$$
T=\widetilde{t}(u) \geq T_{0}
$$

for all $u \in E$ with $\|u\|_{\lambda} \geq R$.

Hence, $\Phi_{\lambda}(u)<0$ for all $u \in E$ with $\|u\|_{\lambda} \geq R$.

Proof of Theorem 1.1 Let $X=E_{\lambda}, Y=Y_{m}$ and $Z=\overline{Z_{m}}$. Clearly, $\Phi(0)=0$ and $\Phi(u)=\Phi(-u)$ due to $\left(F_{4}\right)$. By virtue of Lemma 2.4, Lemma 2.6, Lemma 2.7 and the fountain theorem (Theorem 3.6 [16]), problem (1.1) possesses infinitely many high energy solutions. 
Proof of Corollary 1.2 Let us consider the Hilbert space

$$
H=\left\{u \in H^{1}\left(\mathbb{R}^{3}\right): \int_{\mathbb{R}^{3}}\left(a|\nabla u|^{2}+\lambda \widetilde{V}(x) u^{2}\right) d x<\infty\right\}
$$

endowed with the norm

$$
\|u\|=\left(\int_{\mathbb{R}^{3}}\left(a|\nabla u|^{2}+\lambda \widetilde{V}(x) u^{2}\right) d x\right)^{\frac{1}{2}} .
$$

Let

$$
\Phi(u)=\frac{1}{2} \int_{\mathbb{R}^{3}}\left(a|\nabla u|^{2}+\lambda V(x) u^{2}\right) d x+\frac{b}{4}\left(\int_{\mathbb{R}^{3}}|\nabla u|^{2} d x\right)^{2}-\int_{\mathbb{R}^{3}} F(x, u) d x, \quad \forall u \in H .
$$

Obviously, $\Phi$ is a well-defined class $C^{1}$ functional, and the embedding $H \hookrightarrow L^{s}$ is compact for $2 \leq s<6$ (see the proof of Lemma 2.2). By Lemma 2.4, Lemma 2.6, Lemma 2.7 and the fountain theorem (Theorem 3.6 [16]), problem (1.3) possesses infinitely many high energy solutions.

Remark 2.8 In the next paper, we wish to consider the sign-changing solutions for the biharmonic problem like in $[19,21]$ and so on.

\section{Conclusions}

In this paper, we consider a sequence of high energy weak solutions for the modified quasilinear fourth-order elliptic equation (1.1) under rather weak conditions. We first prove that the energy functional satisfies the Cerami condition in the well-defined Hilbert space and then prove that the fountain theorem holds under the given conditions by a new technique. Our results extend and improve some recent results.

Acknowledgements

This research was supported by the National Science Foundation of China grant 11471187 and 11571197.

Competing interests

The authors declare that they have no competing interests.

Authors' contributions

All authors contributed equally and significantly in writing this article. All authors wrote, read, and approved the final manuscript.

\section{Publisher's Note}

Springer Nature remains neutral with regard to jurisdictional claims in published maps and institutional affiliations.

Received: 21 September 2017 Accepted: 28 March 2018 Published online: 12 April 2018

\section{References}

1. Sun, J.T., Wu, T.: Ground state solutions for an indefinite Kirchhoff type problem with steep potential well. J. Differ. Equ. 256, 1771-1792 (2014)

2. Chipot, M., Lovat, B.: Some remarks on nonlocal elliptic and parabolic problems. Nonlinear Anal. 30(7), 4619-4627 (1997)

3. Fang, X.D., Han, Z.Q.: Existence of a ground state solution for a quasilinear Schrodinger equation. Adv. Nonlinear Stud. $14(4), 941-950(2014)$

4. He, X.M., Qian, A.X., Zou, W.M.: Existence and concentration of positive solutions for quasilinear Schrodinger equations with critical growth. Nonlinearity 26(12), 3137-3168 (2013)

5. Mao, A.M., Chang, H.J.: Kirchhoff type problems in $R^{N}$ with radial potentials and locally Lipschitz functional. Appl. Math. Lett. 62, 49-54 (2016) 
6. Peng, C.Q: The existence and concentration of ground-state solutions for a class of Kirchhoff type problems in $R^{3}$ involving critical Sobolev exponents. Bound. Value Probl. 2017, 64 (2017)

7. Li, Y., Li, F., Shi, J.: Existence of a positive solution to Kirchhoff type problems without compactness conditions. J. Differ Equ. 253, 2285-2294 (2012)

8. Zhang, J., Tang, X.H., Zhang, W.: Existence of multiple solutions of Kirchhoff type equation with sign-changing potential. Appl. Math. Comput. 242, 491-499 (2014)

9. Lazer, A.C., Mckenna, P.J.: Large-amplitude periodic oscillations in suspension bridge: some new connections with nonlinear analysis. SIAM Rev. 32(4), 537-578 (1990)

10. Wu, X.: Existence of nontrivial solutions and high energy solutions for Schrödinger-Kirchhoff-type equations in $\mathbb{R}^{N}$. Nonlinear Anal., Real World Appl. 12, 1278-1287 (2011)

11. Nie, J.J.: Existence and multiplicity of nontrivial solutions for a class of Schrödinger-Kirchhoff-type equations. J. Math. Anal. Appl. 417, 65-79 (2014)

12. Qian, A.X.: Infinitely many sign-changing solutions for a Schrodinger equation. Adv. Differ. Equ. 2011, 39 (2011)

13. Salvatore, A.: Multiple solutions for perturbed elliptic equations in unbounded domains. Adv. Nonlinear Stud. 3, 1-23 (2003)

14. Xu, L.P., Chen, H.B.: Nontrivial solutions for Kirchhoff-type problems with a parameter. J. Math. Anal. Appl. 433, 455-472 (2016)

15. Cheng, B.T., Tang, X.H.: High energy solutions of modified quasilinear fourth-order elliptic equations with sign-changing potential. Comput. Math. Appl. 73, 27-36 (2017)

16. Willem, M.: Minimax Theorems. Birkhäuser, Boston (1996)

17. Rabinowitz, P.H.: Minimax Methods in Critical Point Theory with Applications to Differential Equations. CBMS Reg. Conf. Ser. in Math, vol. 65. Amer. Math. Soc., Providence (1986)

18. Qian, A.X.: Sing-changing solutions for some nonlinear problems with strong resonance. Bound. Value Probl. 2011, 18 (2011)

19. Sun, F.L., Liu, L.S., Wu, Y.H.: Infinitely many sign-changing solutions for a class of biharmonic equation with $p$-Laplacian and Neumann boundary condition. Appl. Math. Lett. 73, 128-135 (2017)

20. Chen, S., Liu, J., Wu, X.: Existence and multiplicity of nontrivial solutions for a class of modified nonlinear fourth-order elliptic equations on $\mathbb{R}^{N}$. Appl. Math. Comput. 248, 593-601 (2014)

21. Mao, A.M., Luan, S.X.: Sign-changing solutions of a class of nonlocal quasilinear elliptic boundary value problems. J. Math. Anal. Appl. 383(1), 239-243 (2011)

\section{Submit your manuscript to a SpringerOpen ${ }^{\circ}$ journal and benefit from:}

- Convenient online submission

- Rigorous peer review

- Open access: articles freely available online

- High visibility within the field

- Retaining the copyright to your article

Submit your next manuscript at $\boldsymbol{\nabla}$ springeropen.com 